\title{
Morphology of a canopy as a preservation structure
}

\author{
I. Markov \& P. Bhiwapurkar \\ School of Architecture, \\ Rensselaer Polytechnic Institute of Technology, USA
}

\begin{abstract}
New technologies have enriched the exploration of architectural designs and ideas. New tools, such as parametric modelling, have enabled architects to investigate designs of "free forms" and shapes of unseen complexity in the past. Rapid prototype models provided new means to examine tectonics and spatial qualities. Yet these "free-morphologies" must recognize constrains of performance needs. This investigation explores a morphology with respect to structural and lighting performance. Structural performances are assessed by finite element analyses by generating stress distributions under typical loads and specific boundary conditions. The evaluation of lighting performance is assessed by evaluating the illumination level on a specified surface area. In the past, build examples show separation of the language of envelope and the language of supporting structure. Here an attempt is made to bring the harmony and blend the two different articulations. The concept is tested in architectural curricula on a real case design scenario of a preservation canopy for Yungang Grottoes in Datong, Shanxi Province, China that has recently been declared a UNESCO world heritage site.
\end{abstract}

Keywords: structure, morphology, free-form, performance, stress, lighting, illumination, daylight, Yungang Grottoes, UNESCO.

\section{Introduction}

Preservation of cultural heritage is attracting attention in today's China. Designs are becoming more sophisticated using digital tools that provide new media in which to generate and explore, contributing to the development of common methods and enriching the means available in the design of contemporary architectonics. The growing presence of these tools is evident not only in the designs of signature buildings, but also in the preservation of historic sites. 
Parametric modelling software enables the creation of forms of previously unseen complexity and combined with 3-D printers allows production of complex physical models for the visualization of ideas, and reveals in-depth tectonics and spatial qualities. Some of the resulting forms are fully free-forms; however, for the complex forms to be realized they must meet their structure and performance criteria.

The conceptual investigation presented here focuses on the structural and lighting evaluation of a free-form canopy as a potential solution for preservation of historic sites. Structural performance is assessed using finite element analysis to generate the stress distribution under typical loads and assigned support conditions. A decrease in the critical stress field is treated as an increase in structural efficacy. The lighting performance is assessed by the level of illumination in the critical plane.

This investigation seeks to achieve harmony and to blend articulation of the envelope with articulation of the structure, presenting a concept for the masscustomization of free-forms. The search for the form is driven by spatial and structural effectiveness. Parametric modelling and structural analysis software were combined to balance creativity with efficiency. The case study is rooted in the Chinese context. It is first tested in the teaching environment and then explored in research studies. The study contributes to challenge how to "meaningful" explore digitally generated forms during the conceptual stage of preservation designs.

\section{Study of the form}

The tectonics of a large volume needed on historic sites is articulated by the generative surface, which can be used to create remarkable spatial conditions. In addition to spatial considerations, the design of the surface form must take into account technical considerations, including structural performance, and illumination conditions. The larger the scale of the project, the greater the importance of these considerations.

A generative surface is tested in a real case design of a space frame canopy for the Yungang Grottoes in Datong, Shanxi Province, China, which has recently been declared a UNESCO World Heritage Site. The grottoes were built around $\mathrm{AD} 450$, and extend for about $1 \mathrm{~km}$ along a mountain ridge, consisting of 53 grottoes that contain approximately 51,000 statues ranging in size from $2 \mathrm{~cm}$ to $17 \mathrm{~m}$. Over the past 1500 years, the statues have been exposed to human damage and erosion by water and wind, resulting in severe decay. The Chinese Government has recently initiated a project to protect the Yungang Grottoes by the construction of a shelter. The focus of the present exploration is the tallest Buddha in Grotto No. 5, which is surrounded by many small Buddhas.

As part of the present study, graduate students from the Department of Architecture, Chinese University of Hong Kong, visited the site and subsequently explored design alternatives for a shelter in the techniques thematic design studio. The mountain ridge that houses the grottoes is $20 \mathrm{~m}$ high, and one 


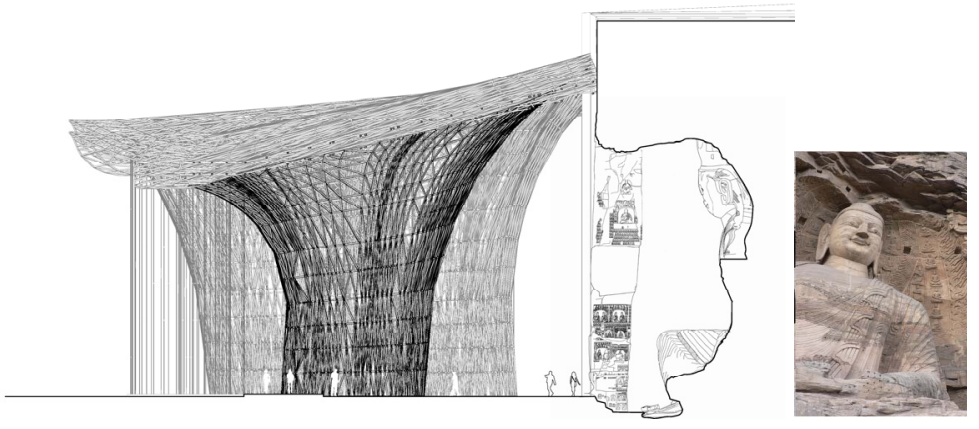

Figure 1: $\quad$ Proposed mushroom canopy to protect Buda statue.

of the requirements is that the shelter it is not supported by this ridge. This requirement is an important consideration because the proposed structure should not read as part of the historic site, as they have no common culture. Moreover, the ridge could not be used to provide reliable support without the need for major intervention. The proposition shown in fig. 1 was selected for further investigation.

The stated intent of maintaining the site in its unattached state while adding a shelter is somewhat self-contradictory, as any new structure would strongly alter the site. Therefore, the response of the new structure to the site conditions was set as a primary goal in architectural articulation. Any shelter would have to enable visitors to sense the site's uniqueness, heritage, religious and spiritual values. A canopy consist of a number of columns yet only three "mushroom" columns are in front of the largest Buda and were part of this investigation as shown in fig. 2. The columns vary in size and shape. The plane of the roof mirrors the form of the ridge, and the columns take the form of pointed arches, thereby highlighting the Buddhas and directing the visitor's observation in their direction.

The structure of the canopy is anticipated as essentially a steel space frame. Such a structure offers light and transparent, yet strong enough system to cover a large volume and respond to large snow loads. Stress fields were used to tune the curves of the canopy plane and shape the parametrically designed "mushroom" columns in order to improve the performance of the entire assembly. A number of areas were identified as having the highest stress concentrations. The curvatures of the plane were iteratively distorted, and at each step the structure was checked to assess whether the critical stress levels had improved or worsened. As the final solution, a more efficient geometry was selected that did not compromise the architectural intent. Figure 2 shows the results after the process was completed for adjusting the curvatures of the canopy plane. 


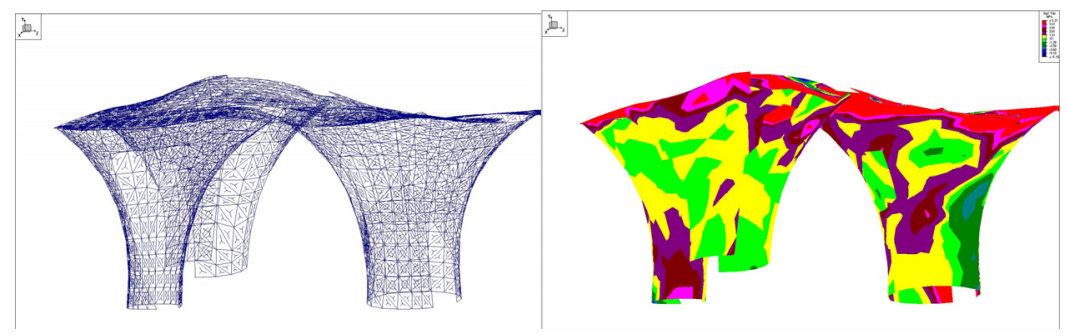

Figure 2: Model mesh and stress distribution after completion of the adjustment process.

In addition to the areas of high stress concentration the figure also reveals areas of low stresses. At the areas of low stress in the structure, the material is not used efficiently and may be removed or replaced by transparent, nonstructural panels. The process of removal of the material subjected to low stresses was carried out manually in an iterative fashion. At each step, those plates subjected to the lowest stresses were removed and the remaining structure was reanalyzed. In subsequent steps, a new set of plates appeared with low stresses, and these were removed in turn. Fig. 2 also shows the approximate shape of the structure after the plates had been removed. It is interesting to observe the locations of voids in the canopy and "mushroom" columns. The voids in the canopy allow for articulation with lighting panels, and openings in the columns might be used as entrances into architectural spaces programmed for services and shops.

The envelope is anticipated as a partly transparent surface that can also contribute to the stability of the overall assemblage yet provide optimal light distribution on the face of the Buda statue. Exploration of lighting conditions was performed in conjunctions with structural exploration.

\section{Daylighting study}

A daylighting study is conducted to investigate the effect of a proposed canopy structure on illumination level inside the cave using three roofing materials; PVC polyester fabric, ethyl tetra fluoroethylene (ETFE) and polytetrafluoroethylene (PTFE). The largest cave of 50'x70'x50' size is selected for this investigation. We focus on the illumination level on the face of the statue. Actual illumination plane is considered in line with the face as shown in fig. 3. Three mushroom columns which support a roof structure stand in front of this cave. The roof and side walls of this structure are central surfaces for this study. The opening of the cave is $50^{\prime}$ tall and 70' wide which is the only source of daylight. This study mainly focuses the illumination levels on a plane of 8'x 8' representing the face. This study is conducted for overcast sky conditions for December 21 . 


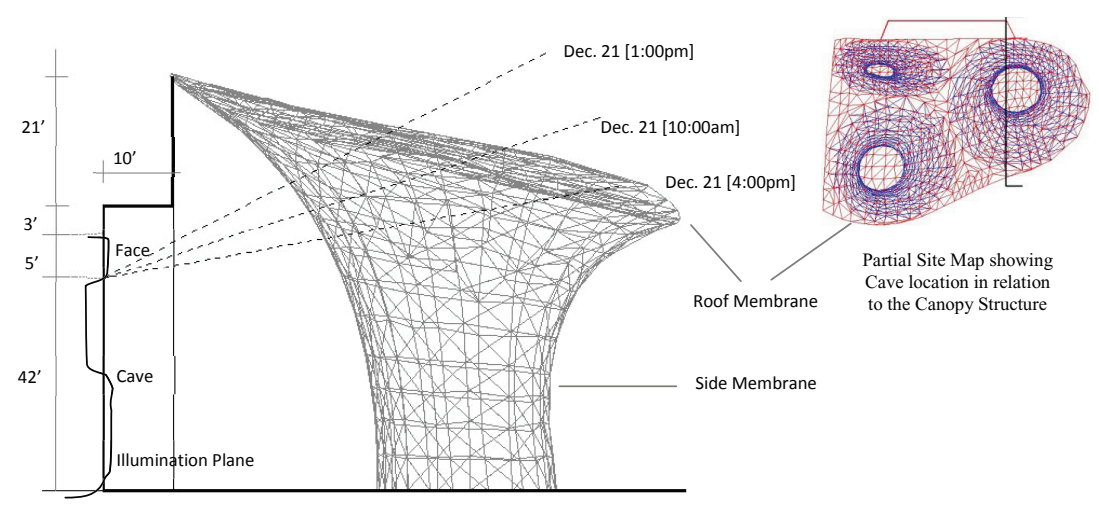

Figure 3: Daylighting study set-up.

The illumination level due to proposed roof/canopy structure is compared with the existing conditions on December 21 at $10 \mathrm{am}, 1 \mathrm{pm}$ and $4 \mathrm{pm}$. The "Baseline" condition is represented by a cave without canopy/roof structure which is similar to the existing condition. First set of investigation is carried out for the effect of time on lighting conditions. The lighting simulations are performed for 10am [Baeline+1], 1pm [Baseline+2] and 4pm [Baseline+3] for overcast conditions as shown in table 1 . The top and side fabric membranes are made up of PVC-polyester fabric and are considered constant for these alternatives. The reflectivity of fabric structure is 0.8 and transmittance is 0.2 .

Second set of investigations focus on materials and replaces the PVCpolyester fabric by ETFE and PTFE. Baseline +5 consider ETFE foil which is lightweight, highly transparent to UV light, is not degraded by sunlight, has better insulation properties than glass, is recyclable and can take up to 400 times its own weight. ETFE reflectivity is 0.95 . Baseline+7 uses a PTFE membrane, which is a versatile, hydrophobic membrane available laminated to various support materials or unsupported. The reflectivity of this material is 0.9 .

The average illumination level for a baseline condition at $10 \mathrm{am}$ is 250.8 Footcandles $(\mathrm{Fc})$ which is the highest amongst all studied configurations. This illumination level is an average of the all calculated illumination values inside the rectangular box shown in table 1. The average illumination level decreases by $69 \%(75.08 \mathrm{Fc})$ at $10 \mathrm{am}$ for Baseline +1 condition when a proposed structural canopy which uses PVC-polyester fabric for roof and side wall is considered in front of the cave. The estimated illumination level at 1:00pm for [Baseline+2] is $72.13 \mathrm{Fc}$. This reduction is $71 \%$ from the baseline conditions. At $4 \mathrm{pm}$ on December 21 on an overcast sky conditions, average illumination level is very low and accounts for $98 \%$ reduction from the baseline condition as shown in table 1 .

The PVC-Polyester fabric material as a source of diffused light is well established however overcast conditions during study period and low solar angle $\left(26.7^{\circ}-17^{\circ}\right)$ are considered a major cause for reduction in natural light for this study. At low sun angles roof structure and importantly mushroom columns 
320 Structural Studies, Repairs and Maintenance of Heritage Architecture XI

Table 1: $\quad$ Comparative average illumination levels on December 21.

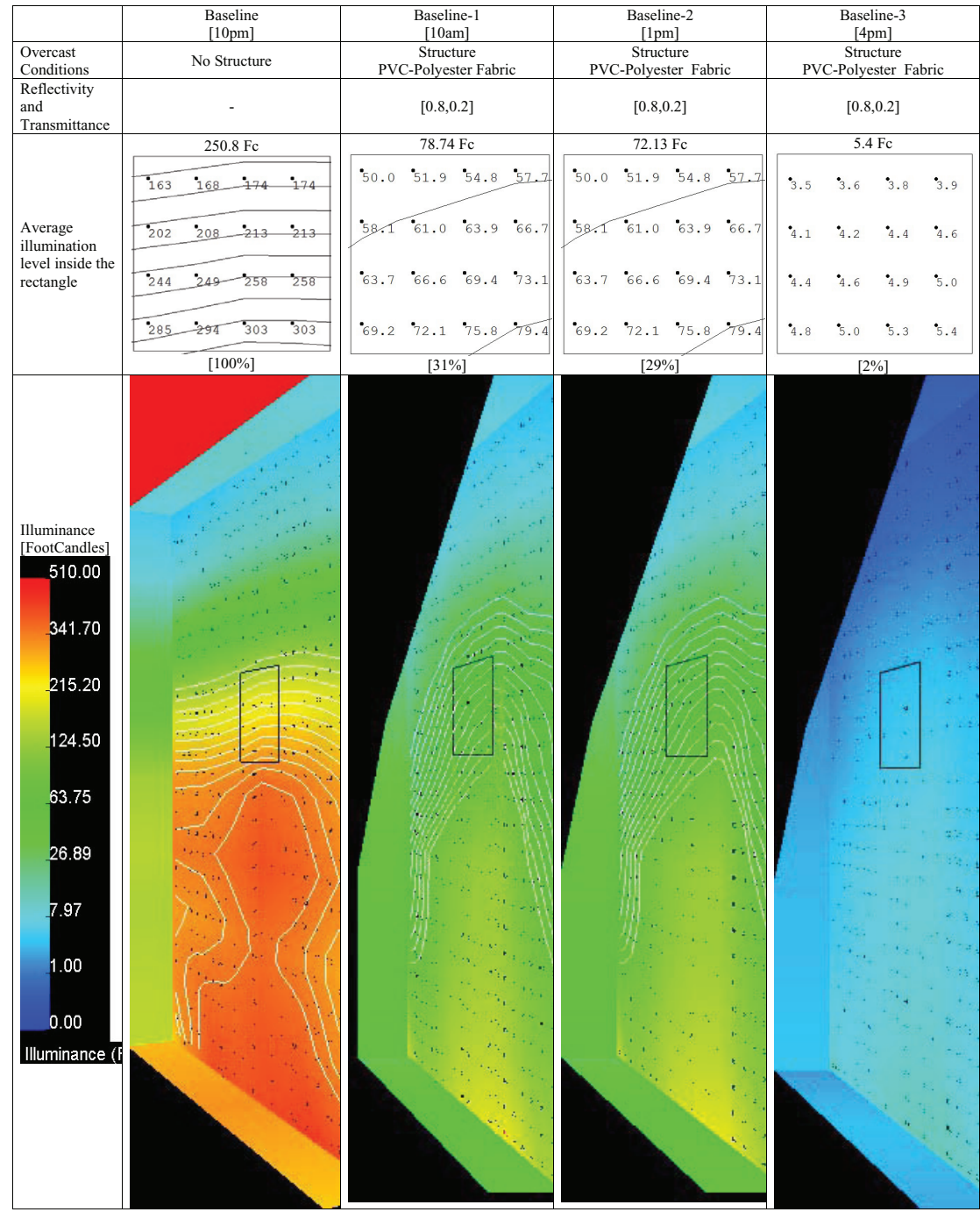

blocks the natural daylight by addition of surface layers between sky and illumination plane. As a result, in Baseline +1 and Baseline +2 illumination levels on columns are much brighter than the receiving vertical plane.

A marginal improvement in illumination level is observed when baseline condition is modified by changing roof and wall membrane properties. The average illumination level of Baseline+5 using ETFE is 5\% (75.86 Fc) above Baseline +4 whereas with increase in lighting condition of Baseline +6 with PTFE is $2 \%(73.62 \mathrm{Fc})$ as shown in table 2 . Although lighting levels are reduced by 
Table 2: Comparative average illumination levels by change in roof and wall material properties.

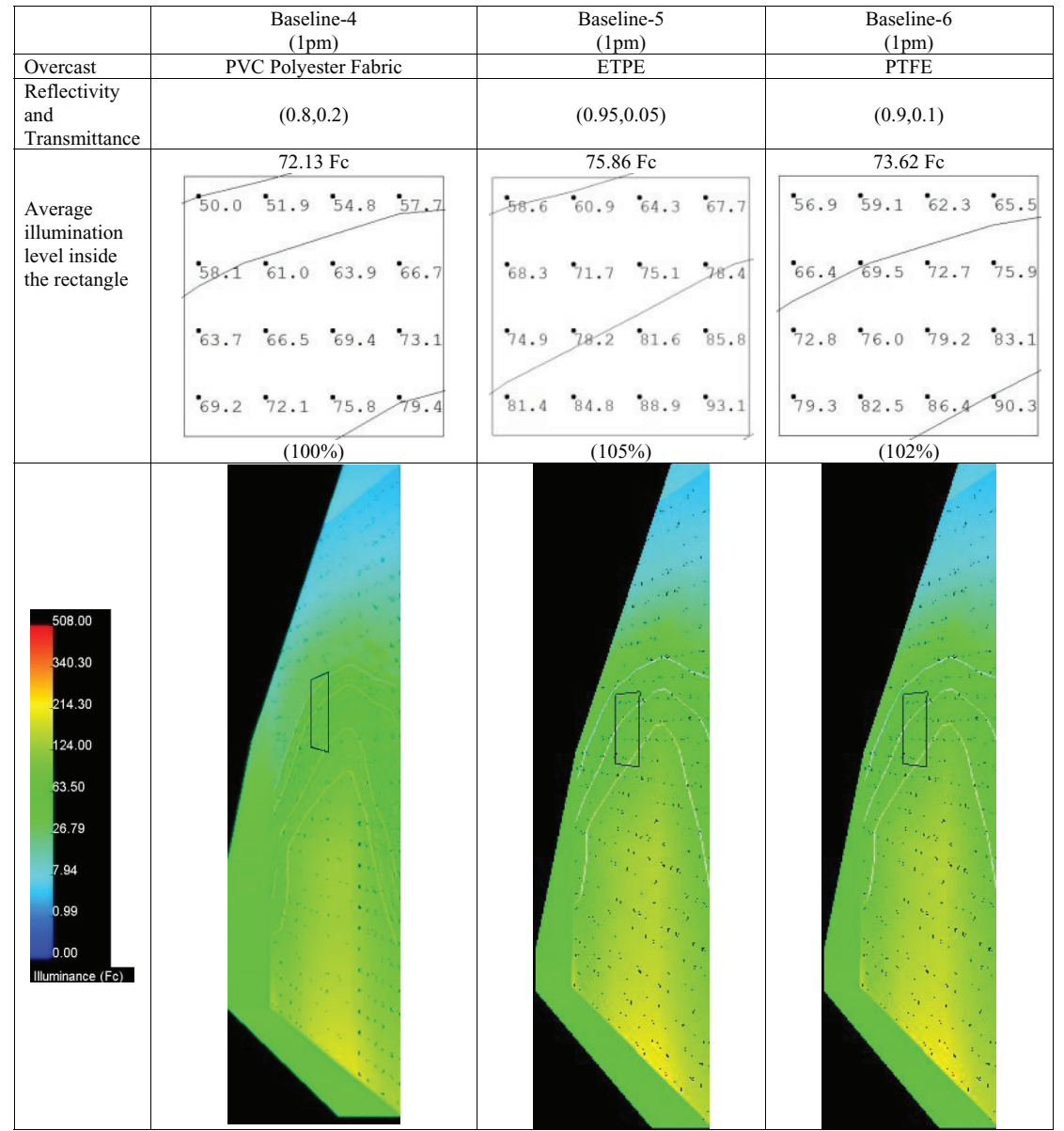

canopy structures, the contrast between calculated highest and lowest illumination level on a vertical is relatively less compared to the baseline conditions thus reduces glare to certain extent.

In addition, the contrast between top and lower portion of an illuminating surface needs attention for minimizing glare. The low solar angle illuminates lower portion of the cave while top portion of the cave remains relatively less illuminated due to the depth of the cave and obstruction by a structural canopy. During clear sky conditions contrast may even be higher however average illumination level increases by $10 \%$. Therefore, a combination of opaque and transparent surfaces in response to the solar geometry may be a useful strategy for achieving uniform lighting conditions inside the cave. 
A major limitation of this study is a simplified rectangular illumination plane considered for investigation of the illumination levels on a spherical face of the statue. Also, the study is performed during overcast sky conditions therefore these results do not take in to account the shading pattern by the structural canopy. The illumination levels will certainly be higher during partly cloudy and clear sky conditions.

However, this study provides evidences that the roof structure can enhance the illumination levels inside the cave by adjusting the material properties according to the solar geometry. The roof and side wall material properties can be used to create uniform illumination levels to minimize glare and need for artificial illumination, if any. The roof membrane surface properties are significant to allow daylight and side membrane can be used for even distribution of diffused light. The curvature of roof and column structure can be a key parameter towards this effect. However, further investigations are required to test the effect of materials and structure to maximize average illumination levels on the top as well as sides of the structure during specific time of the day.

\section{Conclusion}

This study explored the concept of the efficiency of a generative surface for large-volume tectonics proposed for conservation of a heritage site. The form was adjusted slightly to enhance structural performance without compromising the architectural articulation. The adjustment was based on stress field distribution generated by FE analyses. Moreover, those materials not used efficiently in the structure were removed and replaced by transparent panels in the roof or left as voids to enable access into the columns. A design based on a generative surface was tested in a real case design scenario involving the design of a canopy to protect the Yungang Grottoes, China.

Illumination level inside the cave is decreased due to proposed structural canopy by $69 \%, 71 \%$ and $98 \%$ at $10 \mathrm{am}, 1 \mathrm{pm}$ and $4 \mathrm{pm}$ during overcast conditions on December 21 using PVC-Polyester fabric material for roof and side walls. Other lightweight roof material ETFE and PTFE marginally improves the average illumination levels.

\section{References}

[1] Markov, I.," Free-Forms and Technical Efficacy" IASS-IACM 2008 "Spanning Nano to Mega", Cornell University, 28-31 May 2008, Ithaca, NY, USA.

[2] ASAHI Glass Co. http://www.fluon.jp/fluon/english/products/etfe_film/ apply_01.html 